\title{
EXTRAÇÃO DE ESTERÓIDES EM FRUTOS DE Ottonia martiana MIQ., PIPERACEAE, COM GÁS LIQÜEFEITO
}

Miriam Machado Cunico, Obdúlio Gomes Miguel*, Marilis Dallarmi Miguel, João Luiz de Souza Carvalho, Deise Prehs Montrucchio e José Laurentino Ferreira

Departamento de Farmácia, Universidade Federal do Paraná, Av. Prefeito Lothário Meissner, 3400, 80210-170 Curitiba - PR

Juarez Souza de Oliveira

Departamento de Tecnologia Química, Universidade Federal do Paraná, Av. Prefeito Lothário Meissner, 3400, 80210-170

Curitiba - PR

Recebido em 4/9/02; aceito em 9/4/03

\begin{abstract}
EXTRACTION OF STEROLS IN FRUITS OF Ottonia martiana Miq., PIPERACEAE, WITH LIQUEFIED GAS. A new extraction method for sterols was tested in fruits of Ottonia martiana Miq., a shrub belonging to the family Piperaceae, popularly known as "anestésica" due to the use of its roots and aerial parts, in alcoholic preparations, in treatment of odontological problems. For this purpose, a portable extraction equipment was developed, to operate with liquified gases, which showed to be practical, fast and effective, besides being economical and ecological. In the chromatographic analysis of the extracts obtained in this equipment, it was demonstrated the presence of two sterols, isomers of $\beta$-sitosterol and stigmasterol.
\end{abstract}

Keywords: Piperaceae; portable extractor; sterols.

\section{INTRODUÇÃO}

A busca e a intensificação do uso de plantas medicinais pela população mundial têm levado o mercado de fitoterápicos, nutracêuticos e alimentos funcionais a um crescimento surpreendente $^{1}$, o qual tem despertado grande interesse na investigação fitoquímica e no desenvolvimento de novos métodos de extração que apresentem perspectivas para a obtenção de extratos vegetais de modo prático, rápido, eficiente e com baixo custo ambiental e econômico, além de preservar as características naturais das substâncias presentes nos mesmos.

Dentre as várias plantas empregadas na medicina popular brasileira, a Ottonia martiana Miq., Piperaceae, de distribuição característica da Floresta Atlântica e conhecida como "anestésica" pelos nativos do litoral paranaense, destaca-se pelo uso de suas raízes e partes aéreas no tratamento de odontalgias, devido à sua ação anestésica sobre a mucosa bucal, na forma de alcoolatura para a realização de bochechos, ou pela mastigação de fragmentos da planta ${ }^{2}$. Apesar desta propriedade medicinal ser popularmente reconhecida, estudos farmacológicos com a referida planta são inexistentes.

Duas contribuições foram dadas ao estudo fitoquímico desta espécie, resultando no isolamento e identificação das amidas isopiperlonguminina, piperlonguminina e piperovatina, além do sal nitrato de potássio ${ }^{2,3}$. Estas amidas foram isoladas anteriormente das raízes e/ou partes aéreas (caules e frutos) de outras espécies deste gênero, entre outras amidas e terpenos ${ }^{4-10}$.

Sendo assim, este trabalho teve por objetivo testar novo método de extração de esteróides presentes em frutos de $O$. martiana por meio do equipamento portátil extrator para operações com gases liqüiefeitos ${ }^{11}$.

*e-mail: obdulio@ufpr.br

\section{PARTE EXPERIMENTAL}

\section{Instrumentação e material}

As análises cromatográficas por CG foram realizadas no cromatógrafo gasoso Shimadzu CG-14B com coluna DB-1 (100\% dimetilpolisiloxano, polaridade 5, coluna com 0,25 $\mathrm{mm}$ de espessura, de $30 \mathrm{~m}$ de comprimento por $0,53 \mathrm{~mm}$ de diâmetro interno), temperatura inicial $70{ }^{\circ} \mathrm{C} / 3{ }^{\circ} \mathrm{C} \min ^{-1} / 180{ }^{\circ} \mathrm{C} / 10^{\circ} \mathrm{C} \min ^{-1} / 280{ }^{\circ} \mathrm{C}$, split (razão 1:1), purga do septo $3 \mathrm{~mL} \mathrm{~min}^{-1}$, gás de arraste Hidrogênio, vazão de $12 \mathrm{~mL} \mathrm{~min}^{-1}$, solvente metanol e volume injetado de $2 \mu \mathrm{L}$. As análises por CG-EM foram realizadas no cromatógrafo gasoso Varian (EI 70eV), split (1:10), temperatura da fonte $250{ }^{\circ} \mathrm{C}$, coluna VA-5 (30 m x 0,25 mm D.I. x 0,33 mm espessura), temperatura inicial $100{ }^{\circ} \mathrm{C} / 5 \mathrm{~min}$, temperatura programada $100{ }^{\circ} \mathrm{C} / 30{ }^{\circ} \mathrm{C} \mathrm{min} \mathrm{m}^{-1}$ $200^{\circ} \mathrm{C} / 10^{\circ} \mathrm{C} \min ^{-1} / 300{ }^{\circ} \mathrm{C}$, vazão $1,0 \mathrm{~mL} \mathrm{~min}^{-1}$, gás de arraste Hélio, solvente clorofórmio e volume injetado de $20 \mu \mathrm{L}$. As amostras de esteróides utilizadas foram adquiridas da Supelco ( $\beta$-sitosterol 4-7133 e estigmasterol 4-7132). Para as análises por cromatografia em camada delgada foi utilizada como adsorvente a sílica Merck 60 (70-230 mesh). Os solventes utilizados foram P.A. da marca Merck.

A extração foi realizada pelo equipamento portátil extrator para operações com gases liqüefeitos ${ }^{11}$. O gás utilizado no extrator como solvente foi o GLP ("Butane Lighter Gas Extra Purified") da marca Colibri, gás butano refinado para isqueiros.

Segundo Oliveira ${ }^{12}$, o equipamento portátil é constituído de duas partes: corpo A e corpo B (Figura 1). O corpo A, construído em aço inoxidável, possui em uma das extremidades uma válvula (1) de alimentação do solvente (gás liqüefeito), 2 conjuntos de telas (2 e 3), para evitar o entupimento da válvula de alimentação do solvente e problemas de bloqueio e/ou entupimento das válvulas posteriores contidas no corpo B (6 e 9): a dos compartimentos de separação e a que permite a transferência do extrato para o frasco coletor.

O corpo B é constituído de duas câmaras (C e D) com partes tubulares de vidro para visualização da operação de separação das fases (aquosa e orgânica). O conjunto formado pelas câmaras $\mathbf{C}$ e D 


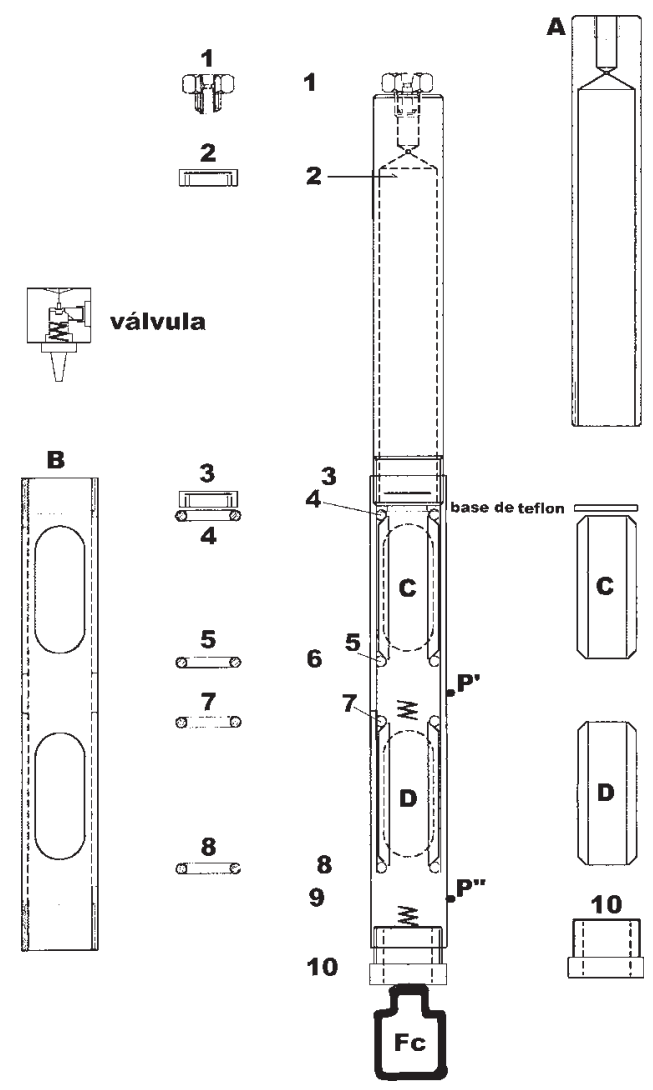

Figura 1. Esquema do equipamento portátil extrator para operações com gases liqüefeitos

comunica-se com o exterior pela válvula 9 , possibilitando a transferência do extrato para um frasco coletor $(\mathrm{Fc})$, por uma agulha, sem contato do mesmo com o meio ambiente.

A montagem do conjunto é feita momentos antes do processo de extração (aproximadamente $5 \mathrm{~min}$ ).

A estanqueidade do conjunto é assegurada pelo uso de anéis de elastômero de borracha nitrílica (4, 5, 7 e 8), colocados entre cada componente do extrator (tubos de vidro e válvulas).

As válvulas, confeccionadas em teflon e aço inoxidável, que em condição de repouso permanecem normalmente fechadas, são operadas pela compressão de um pequeno pino voltado para a parte externa da referida válvula ( $\mathrm{P}$ ' e P”).

\section{Material vegetal}

Frutos de Ottonia martiana Miq., foram coletados em abril de
2000, no município de Guaratuba (encosta da Floresta Atlântica Serra do Mar), PR, à altitude de 20 metros. A identificação da espécie foi realizada pelo botânico Dr. G. Hatschbach do Museu Botânico Municipal (MBM), da Prefeitura de Curitiba, PR, e a exsicata foi depositada no Herbário deste Museu, sob número 259.057.

\section{Obtenção dos extratos vegetais}

Frutos de $O$. martiana foram submetidos a cinco extrações, para completo esgotamento da droga, utilizando-se o equipamento extrator portátil para operações com gases liqüefeitos.

A operação ocorreu alimentando-se o corpo A do extrator com 4,98 g de frutos em pó desta espécie. Em seguida, acoplou-se o corpo A ao corpo B (previamente montado).

A alimentação do solvente (GLP) foi realizada através da válvula superior (1), colocando-se o extrator na posição vertical.

O conjunto extrator (Figura 1) foi mantido na posição vertical, com a base que possui a válvula de alimentação voltada para baixo, o tempo necessário que se tinha estimado para atingir o equilíbrio entre o solvente e a amostra, procedendo-se à coleta de extrato.

Transcorrido o tempo de contato entre o solvente e a amostra, deslocou-se o conjunto extrator, deixando-se a parte que contém a válvula de alimentação (1) voltada para cima, para que o extrato percolasse, passando pelos compartimentos de vidro, pela simples compressão da válvula (P').

A transferência do extrato no compartimento inferior para um frasco coletor deu-se por meio de uma agulha conectada à base da válvula (9) comprimida (P”).

Como a total exaustão do material vegetal, ou seja, daquelas substâncias passíveis de extração pelo solvente, não ocorre em um único ciclo, 5 extrações foram realizadas no mesmo material vegetal, pela realimentação do sistema com outras cargas de solvente.

Cada um dos extratos obtidos foi monitorado por CCD, a fim de verificar se ocorreu completo esgotamento da droga vegetal.

\section{RESULTADOS E DISCUSSÃO}

$\mathrm{O}$ rendimento de cada extrato dos frutos da $O$. martiana obtido por este método foi decrescente, conforme o esperado, demonstrando que as 5 extrações realizadas com o mesmo material vegetal foram suficientes para esgotar completamente a droga vegetal, conforme Tabela 1.

Além disso, verificou-se que o tempo de $2 \mathrm{~h}$ foi considerado suficiente para que o equilíbrio fosse atingido pelo solvente e a amostra vegetal na extração (Tabela 1).

Os extratos monitorados por CCD levaram à análise cromatográfica por CG dos extratos da $1^{\mathrm{a}}, 3^{\mathrm{a}}$ e $5^{\mathrm{a}}$ extrações.

Dentre os componentes detectados por CG nos extratos obtidos (E1 e E3), 2 esteróides foram observados, os quais foram compara-

Tabela 1. Rendimento dos extratos dos frutos da Ottonia martiana Miq., Piperaceae

\begin{tabular}{|c|c|c|c|c|}
\hline Extração dos frutos em pó $(4,98 \mathrm{~g})$ & Massa de GLP utilizada (g) & Tempo (h) & Rendimento (g) & Presença de esteróides \\
\hline $1^{a}$ Extração (E1) & 13,5 & 2 & 0,4102 & $\begin{array}{l}* \text { Isômeros de } \beta \text {-sitosterol } \\
\text { e estigmasterol }\end{array}$ \\
\hline $2^{\mathrm{a}}$ Extração (E2) & 12,8 & 4 & 0,2695 & $\begin{array}{l}* \text { Isômeros de } \beta \text {-sitosterol } \\
\text { e estigmasterol }\end{array}$ \\
\hline $3^{\mathrm{a}}$ Extração (E3) & 10,5 & 2 & 0,2340 & $\begin{array}{l}* \text { Isômeros de } \beta \text {-sitosterol } \\
\text { e estigmasterol }\end{array}$ \\
\hline $4^{a}$ Extração (E4) & 10,9 & 3 & 0,2255 & - \\
\hline $5^{a}$ Extração (E5) & 11,5 & 24 & 0,1744 & - \\
\hline
\end{tabular}

GLP: Colibri ("Butane Lighter Gas Extra Purified”); * Isômeros idênticos 
dos com amostras autênticas dos esteróides estigmasterol e $\beta$ sitosterol. Como os tempos de retenção destes esteróides (30 min< $\mathrm{T}_{\mathrm{R}}<40 \mathrm{~min}$ ) e das amostras autênticas foram similares, realizou-se uma co-injeção do extrato E1 com essas amostras. A análise dos dados cromatográficos após a co-injeção sugeriram tratar-se dos esteróides estigmasterol e $\beta$-sitosterol (Figura 2), cuja identidade não foi confirmada na análise dos dados obtidos por CG-EM dos extratos E1 e E3 (Figura 3).

Os dados obtidos na análise por CG-EM e comparação com os dados de literatura e os da biblioteca eletrônica NIST exibiram alguns fragmentos não compatíveis com aqueles presentes nos espectros de massa dos esteróides estigmasterol e $\beta$-sitosterol, sugerindo tratar-se de isômeros do estigmasterol e $\beta$-sitosterol.

$\mathrm{O}$ pico intenso que aparece nos cromatogramas da Figura $2\left(\mathrm{t}_{\mathrm{R}}\right.$ $\sim 19 \mathrm{~min}$ ), refere-se a um resíduo do solvente utilizado na análise cromatográfica.

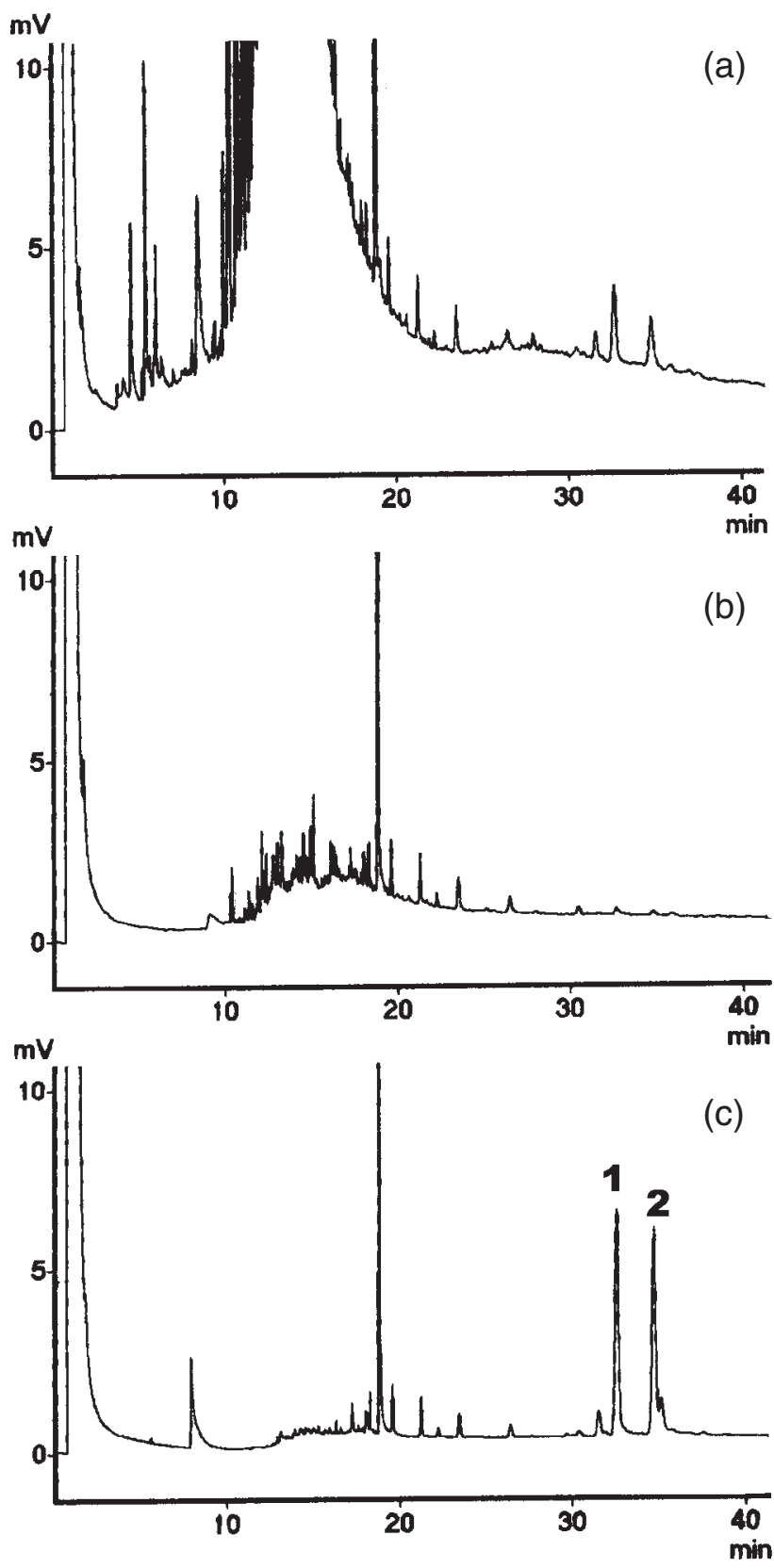

Figura 2. Cromatogramas $(G C): a-E 1 ; b-E 3 ; c-$ Co-Injeção: $E 1+$ Amostras autênticas; (estigmasterol- 1 e $\beta$ sitosterol - 2)

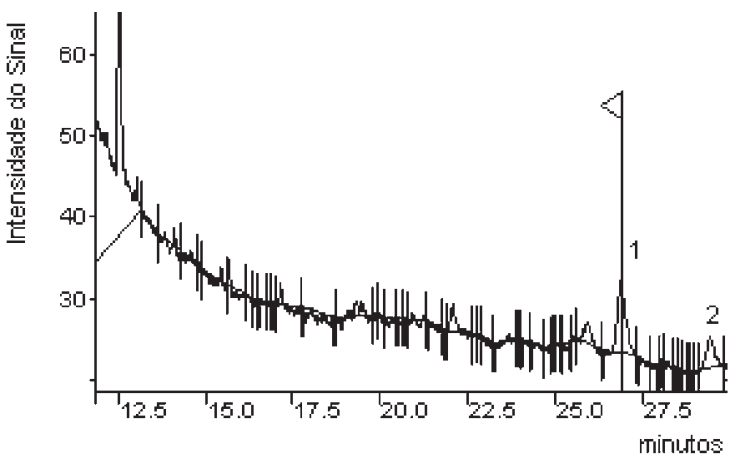

(a)
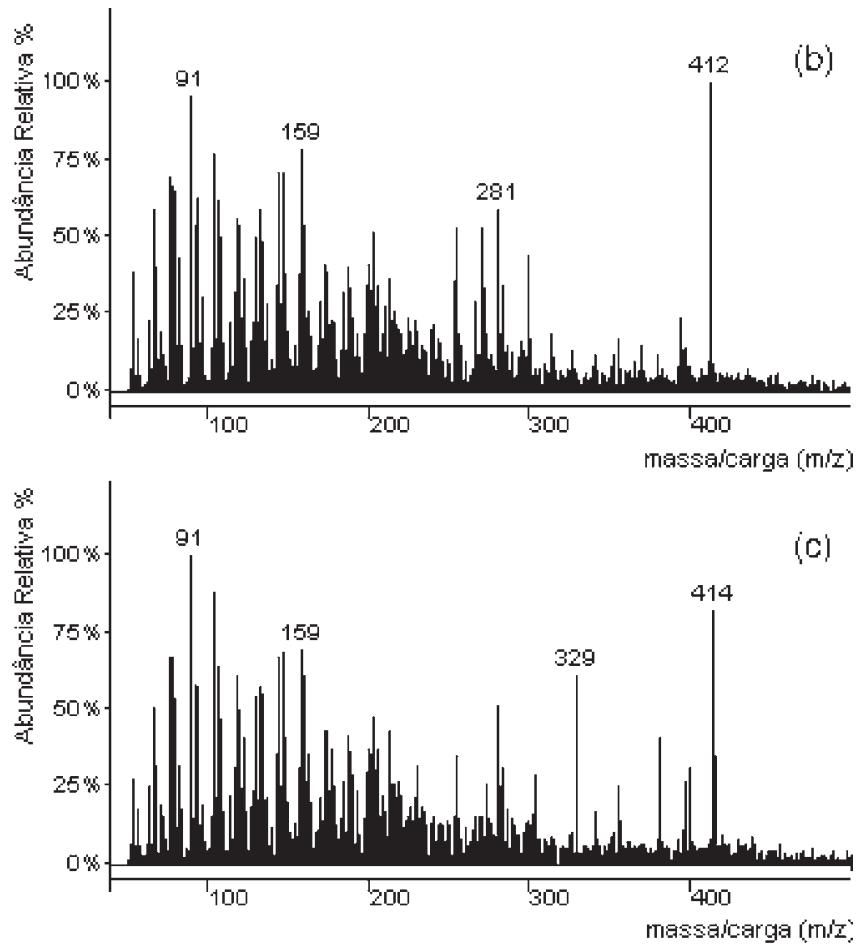

Figura 3 - Dados da análise por CG-EM do extrato E1 dos frutos da Ottonia martiana. a - cromatograma do extrato E1; $b$ - espectro de massa: isômero do estigmasterol (1); c - espectro de massa: isômero do $\beta$-sitosterol (2)

Os outros picos observados nos cromatogramas encontram-se em fase de identificação, visto serem prováveis óleos essenciais e óleos graxos.

\section{CONCLUSÃO}

O extrator portátil permitiu a extração de esteróides presentes nos frutos de $O$. martiana (isômeros dos compostos estigmasterol e $\beta$-sitosterol), os quais foram detectados nos extratos E1 e E3 por CCD e CG, e caracterizados por CG-EM e comparação com dados de literatura e padrões das bibliotecas eletrônicas (Wiley e NIST).

Sendo assim, este método de extração de esteróides por equipamento portátil extrator para operação com gases liqüefeitos apresenta perspectivas para a obtenção de extratos de plantas com praticidade e rapidez, a um custo ambiental e econômico baixo, devido ao descarte do solvente e ao tempo de extração da amostra.

Entretanto, mais estudos deverão ser realizados com este equipamento extrator utilizando-se solventes mais polares (outros gases mais polares), a fim de aumentar a seletividade dos compostos possibilitando, desta forma, o fracionamento destes extratos com maior eficácia e permitindo a comparação deste método com outros já existentes. 


\section{AGRADECIMENTOS}

Ao CNPq pelo suporte financeiro, ao Dr. G. Hatschbach do Museu Botânico Municipal da Prefeitura de Curitiba, pela identificação da espécie vegetal e ao coletor O. S. Ribas do MBM ( $\left.n^{\circ} 3633\right)$, pela obtenção do material vegetal estudado.

\section{REFERÊNCIAS}

1. Miguel, M. D.; Miguel, O. G.; Desenvolvimento de fitoterápicos, São Paulo: Robe Editorial, 1999, p. 11-15.

2. Lopes, M.; Dissertação de Mestrado, Universidade Federal do Paraná, Brasil, 1989.
3. Cunico, M. M.; Dissertação de Mestrado, Universidade Federal do Paraná, Brasil, 2001.

4. Dunstan, W. R.; Garnett, H.; J. Chem. Soc. 1895, 67, 94.

5. Giesbrecht, A. M.; Alvarenga, M. A.; Gottlieb, O. R.; Gottlieb, H. R.; Planta Med. 1981, 43, 375.

6. Hänsel, R.; Leuschke, A.; Bohlmann, F.; Planta Med. 1980, 40, 161.

7. Costa, S. S.; Mors, W. B.; Phytochemistry 1981, 20, 1305.

8. Makapugay, H.; Soejarto, D. D.; Kinghorn, A. D.; Bordas, E.; J. Ethnopharmacol. 1983, 7, 235.

9. Moreira, D. L.; Kaplan, M.; Guimarães, A.; Journal of Essential Oil Research 1997, 9, 565.

10. Pinder, A. R.; Price, S. J.; J. Chem. Soc. 1967, C, 2598.

11. Oliveira, J. S.; Br PI 9804199-1 1998.

12. Oliveira, J. S.; Dissertação de Mestrado, Universidade Federal do Paraná, Brasil, 1997. 